\title{
Mitochondrial transplantation: applications for pediatric patients with congenital heart disease
}

\author{
Sitaram M. Emani, James D. McCully \\ Department of Cardiovascular Surgery, Boston Children's Hospital, Boston, USA \\ Contributions: (I) Conception and design: All authors; (II) Administrative support: All authors; (III) Provision of study materials or patients: All \\ authors; (IV) Collection and assembly of data: All authors; (V) Data analysis and interpretation: All authors; (VI) Manuscript writing: All authors; (VII) \\ Final approval of manuscript: All authors. \\ Correspondence to: Sitaram M. Emani, MD. 300 Longwood Ave., Boston, MA 02115, USA. Email: Sitaram.Emani@cardio.chboston.org.
}

\begin{abstract}
Mitochondrial transplantation refers to transplantation of respiratory competent mitochondria from healthy tissue into tissues injured by ischemia and reperfusion. This technique has been utilized for recovery of myocardial dysfunction in pediatric patients. The preclinical experience and initial patient experience with this technique are reviewed in this article. Initial experience is with pediatric patients undergoing extracorporeal membrane oxygenation support following myocardial ischemia and reperfusion. The initial pediatric experience suggests low side effect profile with favorable efficacy in a small group of patients.
\end{abstract}

Keywords: Mitochondria; extracorporeal membrane oxygenation (ECMO)

Submitted Nov 26, 2017. Accepted for publication Jan 31, 2018.

doi: 10.21037/tp.2018.02.02

View this article at: http://dx.doi.org/10.21037/tp.2018.02.02

\section{Introduction}

Myocardial ischemia and reperfusion injury in adults is typically associated with acquired coronary artery disease, however, pediatric patients are also at risk for this condition following cardiac surgical procedures, or in the setting of hypercoagulability mediated thrombosis of the coronary vasculature. Ischemia and reperfusion injury has a profound impact on the myocardium and significantly contributes to morbidity and mortality (1-8). Myocardial ischemia significantly damages mitochondrial structure and function and these decrements persist into reperfusion to severely compromise post-ischemic functional recovery and cellular viability (1-8). Currently available pharmacologic and genetic tools and procedural techniques intended to targeted mediators of ischemia and reperfusion injury have for the most part had only limited success (9-14). In clinical practice, cardiac function is typically stimulated by inotropic agents such as beta-adrenergic agonists and phosphodiesterase inhibitors, but these agents do not rectify the underlying abnormalities in mitochondrial structure and function and have detrimental effects upon myocardial cellular energetics, myocardial cellular viability and receptor desensitization.

Recently, we have pioneered a novel therapy based on mitochondrial transplantation (15-18). This procedure uses replacement of native mitochondria with viable, respirationcompetent mitochondria isolated from non-ischemic autologous tissue to overcome the many deleterious effects of ischemia/reperfusion injury on native mitochondria (15-18). This review summarizes some of the preclinical and clinical experience with mitochondrial transplantation for myocardial ischemia and reperfusion injury.

\section{Mitochondrial transplantation in preclinical models}

We have validated the efficacy of mitochondrial transplantation in a series of in vitro, isolated perfused and in vivo perfused animal heart models using ischemia/ reperfusion protocols (15-18). Our studies have demonstrated that autologous mitochondria isolated form 
the patient's own body and then directly injected into the ischemic myocardium significantly ameliorates ischemia/ reperfusion injury and enhances myocardial cellular viability and post-ischemic functional recovery $(15,16,18)$.

The transplanted mitochondria act both extra- and intracellularly. The transplanted mitochondria immediately increase myocardial ATP levels, extra-cellularly and ATP synthesis, intra-cellularly and rapidly alter the myocardial proteome to upregulate pathways for energy generation and cellular respiration and upregulate cardioprotective cytokines $(16,17)$. These cytokines are associated with reduced cardiomyocyte apoptosis and enhanced functional cardiac recovery and cardiac remodeling independent of cardiac myocyte regeneration (16). The mitochondria are rapidly internalized (2.5-60 min) into myocardial cells by actin-dependent endocytosis where they up-regulate cellular function and repair damaged mitochondrial DNA $(17,19)$. The transplanted, mitochondria maintain viability and function for at least 28 days and have no pro-arrhythmic inflammatory, immune or auto-immune response $(16,18,20)$.

\section{Pediatric applications of mitochondrial transplantation}

Several clinical scenarios have been identified in which myocardial ischemia and reperfusion injury may contribute to myocardial dysfunction. Surgical manipulation of the coronary arteries is necessary in many of these conditions including transposition of the great arteries, anomalous origin of the coronary artery from the pulmonary artery or inappropriate sinus of Valsalva. Coronary artery occlusion can be associated with other procedures performed in the vicinity of the coronary artery (aortic, mitral, or pulmonary valve replacement). These surgical procedures may result in either direct mechanical compression of the coronary artery (kinking or external compression) or predispose to intravascular thrombosis due to altered flow dynamics and resultant ischemia. The clinical presentation of acute coronary artery insufficiency in pediatric patients is typically immediate and dramatic. Frequently intraoperative sustained coronary artery injury leads to inability to separate from cardiopulmonary bypass. Myocardial dysfunction may also be temporarily supported by aggressive inotropic support but eventually leads to hemodynamic collapse. Early recognition of coronary artery occlusion requires high degree of clinical suspicion as well as confirmatory vascular imaging, typically with angiography. Early intervention for relief of obstruction and revascularization is critical to prevent development of irreversible myocardial injury or infarction. Revascularization is typically performed immediately after the diagnosis is confirmed, and may be performed through catheter-based intervention or surgical approaches. However, despite aggressive strategy for early revascularization, these patients continue to demonstrate significant myocardial dysfunction that persists for days to weeks after revascularization and reperfusion event. This form of ischemia reperfusion injury surrounding an acute identifiable event is an appropriate target for mitochondrial transplantation.

Conditions leading to coronary artery occlusion with subsequent revascularization are deemed the most suitable candidates for the targeted therapy. Although rare in children, acquired stenosis of coronary vasculature is associated with conditions such as Kawasaki disease and congenital hyperlipidemia. However, occlusion of the coronary arteries tends to be gradual in these conditions, and the degree of collateral vascular development determines the degree of myocardial ischemia. At this time, these patients have not been considered as appropriate candidates for mitochondrial transplantation, as traditional mechanisms of ischemia and reperfusion injury may not be applicable. Patients suffering from cardiopulmonary resuscitation (CPR) may experience varying degrees of myocardial ischemia as a result of altered cardiac output. Depending upon the duration of CPR, these patients may also suffer some degree of ischemia and reperfusion injury following reestablishment of circulation. Mitochondrial dysfunction may result in depressed myocardial function following such resuscitation, and may be ameliorated by mitochondrial transplantation.

Myocardial ischemia is induced in patients undergoing cardiopulmonary bypass for repair of congenital heart disease. Controlled myocardial ischemia is produced by interruption of coronary artery blood flow by placement of the aortic cross-clamp and delivery of cardioplegia to achieve diastolic myocardial arrest. Although cardioplegia arrest is eventually associated with myocardial functional recovery, varying degrees of mitochondrial dysfunction have been demonstrated in patients with prolonged duration of ischemia. In such patients, mitochondrial transplantation may play a role in reducing the degree of ischemia/ reperfusion-related myocardial injury.

\section{Mitochondrial isolation}

Our studies have demonstrated that to allow for clinical 


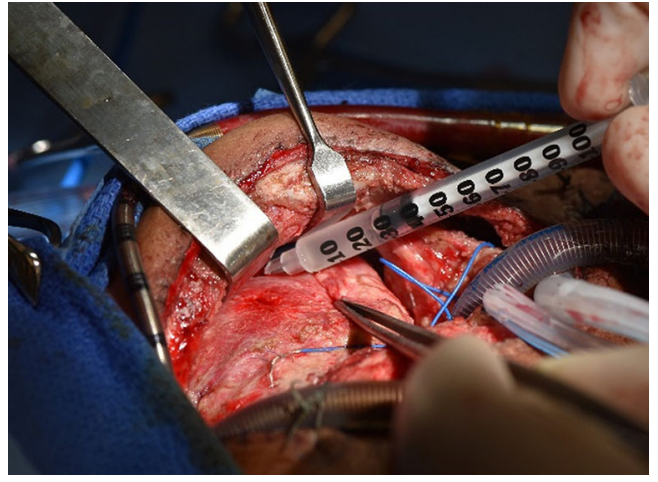

Figure 1 Mitochondrial transplantation procedure involves injection of harvested mitochondria into dysfunctional myocardium.

efficacy, mitochondrial transplantation requires freshly isolated, viable mitochondria. Non-viable mitochondria, previously frozen mitochondria, mitochondrial fractions (proteins, complex I-V), mitochondrial DNA and RNA, and exogenous ATP or ADP do not provide cardioprotection (15). No difference in efficacy is observed using total, interfibrillar or sub-sarcolemmal mitochondria (15).

To allow for clinical application, we have developed a rapid methodology for the isolation of autologous mitochondria and have established Good Manufacturing Practice (GMP) and quality control and quality assurance standards to ensure consistency and quality of mitochondria isolation $(21,22)$.

Mitochondrial isolation can be performed in $<30$ minutes, and can be performed near-patient. The equipment and reagents necessary are widely available in most laboratory settings and the reagents utilized are approved for clinical usage (21). In brief two small pieces of skeletal muscle tissue $(<0.1$ gram) are obtained using a \#6 biopsy punch (6 $\mathrm{mm}$ diameter, $3 \mathrm{~mm}$ depth). We have isolated mitochondria from the pectoralis major muscle and abdominal rectus muscle based on surgical entry point (minimal thoracotomy, sternotomy). The tissue is homogenized in a volume of $5 \mathrm{~mL}$ of isolation buffer (300 $\mathrm{mmol} / \mathrm{L}$ sucrose, $10 \mathrm{mmol} / \mathrm{L}$ HEPES-KOH, $1 \mathrm{mmol} / \mathrm{L}$ EGTA-KOH, $\mathrm{pH}$ 7.4) and then treated to 10 minutes of Subtilisin A enzymatic digestion on ice. The digested tissue is then filtered through a series of filters pre-wetted with isolation buffer, and the mitochondria are subsequently precipitated by centrifugation at $9 \times \mathrm{G}$ for 5 minutes at $4{ }^{\circ} \mathrm{C}$. The method for isolation of mitochondria and quality control standards are available $(21,22)$.
Mitochondrial number, viability and purity are assessed in all mitochondrial preparations using an aliquot of the isolated mitochondria. Mitochondrial number and size are determined by particle size analysis and light microscopy using fluorescent mitochondrial specific labels MitoTracker Red CMXRos (Invitrogen, Carlsbad, CA, USA) which labels only viable mitochondria maintaining membrane potential followed by MitoTracker Green FM, which labels all mitochondria independent of membrane potential and then by transmission electron microscopy $(15-17,19)$. Mitochondrial viability is determined by oxygen consumption rate using a Clark-type electrode and fluorescent probe analysis $(15,17,21,22)$. Please note that labeled mitochondria are not used for transplantation to patients.

\section{Timing considerations}

In preclinical studies, mitochondrial transplantation was performed immediately prior to reperfusion of the myocardium (23). In clinical scenarios of prolonged intraoperative cross-clamp time, mitochondrial transplantation just prior to cross-clamp removal would be consistent with the timing outlined in animal models. However, in many clinical situations of coronary artery obstruction, revascularization is promptly performed, and mitochondrial transplantation prior to reperfusion may not be feasible. However, the salutary effects of mitochondrial transplantation may be achieved even if transplantation is performed following reperfusion.

\section{Method of delivery}

Once isolated, the mitochondria are immediately used for mitochondrial transplantation. The isolated mitochondria can be delivered either by direct injection into the ischemic region of the myocardium or by vascular infusion. Mitochondrial transplantation has currently been performed by direct injection into tissue bed. Mitochondria are injected into the myocardium using a tuberculin syringe with a 28 or 32-gauge needle (Figure 1). Mitochondria are delivered in $100 \mu \mathrm{L}$ injections. There is no need for suturing as there is no flush-back using this volume $(15,16,18)$. Direct Injection of mitochondria provides localized distribution of mitochondria (20). This is the current validated method of delivery utilized in our initial preclinical work. One advantage of direct injection is ability to target specific regions of the myocardium that demonstrate regional 
dysfunction. A drawback is that injection to the posterior of the heart requires manipulation of the heart.

Alternatively, mitochondria can be delivered by antegrade intravascular delivery (20). To allow for intravascular access to the left co-arterial system, the artery is dissected, exposed and accessed with a $5 \mathrm{~F}$ arterial access sheath. Mitochondria (in respiration buffer) are then injected antegrade, into the artery as a bolus (20). Vascular delivery of mitochondria results in the rapid and widespread distribution of mitochondria throughout the tissue (20). Mitochondrial uptake into the myocardium by vascular delivery, based on ${ }^{18} \mathrm{~F}$ washout indicates that the majority of ${ }^{18} \mathrm{~F}$-Rhodamine 6 G-labeled mitochondria remain in the hearts during reperfusion. Myocardial uptake of mitochondria following intracoronary delivery has been confirmed by histology and transmission electron microscopy using immuno-gold labeling (20).

Intravascular delivery may be performed by several methods including direct intracoronary injection via selective coronary artery catheterization or aortic root injection with occlusion of the ascending aorta. Delivery into the aortic root may be preferred for mitochondrial transplantation intra-operatively following prolonged cardiopulmonary bypass prior to cross-clamp removal as it provides global distribution of mitochondrial transfer. On the other hand, transcatheter selective coronary infusion may be applicable for patients undergoing transcatheter revascularization in the catheterization laboratory.

\section{Dosing considerations}

Studies in animal models of ischemia-reperfusion have been performed to determine the optimal dose of autologous mitochondria for cardioprotection by direct injection $(15,16,18,24)$. Mitochondrial dose of approximately $2 \times 10^{5}$ mitochondria per gram of tissue wet weight was determined to be an optimal dose in the studies, and has been used for initial pilot trials in humans. In a $4 \mathrm{~kg}$ neonate, this corresponds to a dose of approximately $1 \times 10^{9}$ mitochondria. However, dose escalation studies in a controlled clinical trial will be necessary to validate these results. The total volume of mitochondria is typically re-suspended in $1 \mathrm{~mL}$ of buffer and delivered by 10 to 15 direct epicardial injections.

\section{First-in-man pilot clinical study of autologous mitochondrial transplantation to improve cardiac function}

The initial clinical application of mitochondrial transplantation in humans occurred in pediatric patients suffering from myocardial ischemia reperfusion injury following coronary artery occlusion and revascularization (23). Specifically, patients with ischemia reperfusion injury who required institution of extracorporeal membrane oxygenation (ECMO) support by central cannulation (through sternotomy approach) were selected for mitochondrial transplantation. Firstly, patients with ventricular dysfunction who require ECMO support have extremely poor prognosis based upon historical data, and thus were felt to be the subgroup of patients that would most greatly benefit from this therapy. ECMO support for more than 72 hours is a grave prognostic indicator, with overall survival only $40 \%$ at Boston Children's Hospital and $<30 \%$ reported in the literature (25). Secondly, the presence of mechanical circulatory support allows for safety margin if complications such as arrhythmias or further myocardial injury were to be encountered as a result of transplantation. Finally, in patients who undergo central cannulation, the epicardium and skeletal muscle are readily accessible through the same incision, thus preventing the need for additional incisions for muscle biopsy or mitochondrial delivery.

Since mitochondrial harvest and isolation can be performed within 20-30 minutes during the same procedure and involves minimal manipulation of muscle tissue, the procedure is classified by the FDA as a "Same Procedure Exception". The initial transplantation procedures were performed under an "Innovative Therapies" designation from the Boston Children's Hospital Institutional Review Board (IRB). Review of the proposed therapy was provided by two independent physicians who were not involved with the patients' care. Families were extensively counseled regarding the novelty of the procedure and its potential complications. Separate Innovative Therapies consent forms were signed. Following initial demonstration of safety of mitochondrial transplantation, a prospective observational pilot study was designed and has been approved by the IRB for further enrollment (23).

Patients were considered for mitochondrial transplantation in the initial clinical experience had to meet certain strict inclusion criteria: (I) recent cardiac surgical procedure preceding coronary ischemia; (II) acute onset of coronary artery obstruction, documented by angiography or other imaging studies; (III) surgical or transcatheter revascularization of coronary obstruction within 48 hours of documentation of coronary artery occlusion; (IV) mechanical support with ECMO through 
central cannulation; (V) documented myocardial systolic dysfunction by echocardiogram following reperfusion. Patients were excluded if they had known history of mitochondrial disorder, if they had sustained prolonged ischemia leading to irreversible myocardial infarction, or if systolic function by echocardiogram had improved following reperfusion event (23).

Mitochondrial transplantation was performed in the intensive care unit or the operating theater. The temporary barrier overlying the mediastinum was removed, and the chest cavity was evacuated of hematoma. The rectus abdominis muscle, accessed at the inferior aspect of the sternotomy incision was exposed and assessed for viability of the skeletal muscle. Alternative sources of skeletal muscle include sternohyoid, pectoralis, or sternocleidomastoid muscles which are also accessible through this incision. Approximately $6 \mathrm{~mm} \times 6 \mathrm{~mm}$ sample of muscle was removed for mitochondrial isolation. Despite full anticoagulation, none of the patients experienced bleeding at the site of skeletal muscle harvest (23).

Mitochondria were isolated within the surgical environment as described above. Locations of epicardial injections were chosen based upon epicardial echocardiography determination of hypokinetic regions corresponding with areas of hibernating myocardium. Mitochondria were then injected into the myocardium using a 28-gauge needle. Between 50 and $100 \mu \mathrm{L}$ of mitochondrial suspension were delivered at each injection site, and approximately 10 to 15 injections were performed per patient. This corresponded with the dose of approximately $1 \times 10^{9}$ mitochondria per patient.

Epicardial injections must be performed so as to avoid extravasation of infusate. This is performed by gentle infusion of mitochondria with each injection over the course of approximately 30 seconds for each injection. Gentle pressure is placed at the site of needle entry to avoid extravasation. Despite systemic anticoagulation, bleeding from the myocardial injection sites is uncommon. Following mitochondrial transplantation and epicardial echocardiogram is immediately performed to exclude development of intra-myocardial hematoma.

None of the patients experienced adverse short-term complications related to mitochondrial injection (e.g., arrhythmia, intra-myocardial hematoma, or scarring). In 4 of the 5 initial patients, recovery of heart function was seen, allowing separation from ECMO. The first patient in this series had sustained coronary artery occlusion following arterial switch operation at an outside institution and was supported by ECMO for 2 weeks prior to transfer to our institution. Myocardial function did not improve despite revascularization, and mitochondrial transplantation was applied as salvage therapy. This patient eventually succumbed to complications of renal failure on ECMO and on autopsy was found to have a transmural infarction in the myocardium. Given the prolonged duration of ischemia in this patient, recovery with mitochondrial transplantation was unlikely. For subsequent patients, mitochondrial transplantation was performed within 48 hours of reperfusion event. Improvement in myocardial function was typically seen within 24 to 48 hours following mitochondrial transplantation. The median time to decannulation following reperfusion in this cohort was 4 days (23).

Given the small number of patients and lack of control group, it was not possible to attribute improvement in ventricular function to mitochondrial transplantation, although from historical data suggests grim prognosis for patients who require ECMO support following postoperative coronary artery occlusion. Properly designed prospective, randomized trials will be necessary to determine if mitochondrial transplantation is superior to current standard of care.

\section{Future directions}

Mitochondrial transplantation may be expanded to treat other forms of myocardial ischemia reperfusion injury if randomized trials in pediatric patients demonstrate positive results. Adjunctive treatment during coronary artery revascularization in adult patients is an obvious application. In addition, it may be beneficial for treatment of various other conditions in other organ systems that result from ischemia-reperfusion injury (26-29). Preliminary data suggests that it may enhance recover following stroke and liver ischemia-reperfusion $(28,29)$.

\section{Acknowledgements}

Support provided by Donald Bulens and Lynne Capozzi Foundation.

\section{Footnote}

Conflicts of Interest: The authors have no conflicts of interest to declare. 


\section{References}

1. Tsukube T, McCully JD, Metz KR, et al. Amelioration of ischemic calcium overload correlates with high-energy phosphates in senescent myocardium. Am J Physiol 1997;273:H418-25.

2. Levitsky S, Laurikka J, Stewart RD, et al. Mitochondrial DNA deletions in coronary artery bypass grafting patients. Eur J Cardiothorac Surg 2003;24:777-84.

3. McCully JD, Bhasin MK, Daly C, et al. Transcriptomic and proteomic analysis of global ischemia and cardioprotection in the rabbit heart. Physiol Genomics 2009;38:125-37.

4. McCully JD, Wakiyama H, Hsieh YJ, et al. Differential contribution of necrosis and apoptosis in myocardial ischemia-reperfusion injury. Am J Physiol Heart Circ Physiol 2004;286:H1923-35.

5. McCully JD, Toyoda Y, Wakiyama H, et al. Age- and gender-related differences in ischemia/reperfusion injury and cardioprotection: effects of diazoxide. Ann Thorac Surg 2006;82:117-23.

6. Rousou AJ, Ericsson M, Federman M, et al. Opening of mitochondrial KATP channels enhances cardioprotection through the modulation of mitochondrial matrix volume, calcium accumulation, and respiration. Am J Physiol Heart Circ Physiol 2004;287:H1967-76.

7. McCully JD, Rousou AJ, Parker RA, et al. Age- and gender-related differences in mitochondrial oxygen consumption and calcium with cardioplegia and diazoxide. Ann Thorac Surg 2007;83:1102-9.

8. Lesnefsky EJ, Chen Q, Slabe TJ, et al. Ischemia, rather than reperfusion, inhibits respiration through cytochrome oxidase in the isolated, perfused rabbit heart: role of cardiolipin. Am J Physiol Heart Circ Physiol 2004;287:H258-67.

9. Klein HH, Pich S, Lindert S, et al. Treatment of reperfusion injury with intracoronary calcium channel antagonists and reduced coronary free calcium concentration in regionally ischemic, reperfused porcine hearts. J Am Coll Cardiol 1989;13:1395-401.

10. Carry MM, Mrak RE, Murphy ML, et al. Reperfusion injury in ischemic myocardium: protective effects of ruthenium red and of nitroprusside. Am J Cardiovasc Pathol 1989;2:335-44.

11. Gumina RJ, Buerger E, Eickmeier C, et al. Inhibition of the $\mathrm{Na}(+) / \mathrm{H}(+)$ exchanger confers greater cardioprotection against 90 minutes of myocardial ischemia than ischemic preconditioning in dogs. Circulation 1999;100:2519-26; discussion 2469-72.

12. Boden WE, van Gilst WH, Scheldewaert RG, et al. Diltiazem in acute myocardial infarction treated with thrombolytic agents: a randomised placebo-controlled trial. Incomplete Infarction Trial of European Research Collaborators Evaluating Prognosis post-Thrombolysis (INTERCEPT). Lancet 2000;355:1751-6.

13. Théroux P, Chaitman BR, Danchin N, et al. Inhibition of the sodium-hydrogen exchanger with cariporide to prevent myocardial infarction in high-risk ischemic situations. Main results of the GUARDIAN trial. Guard during ischemia against necrosis (GUARDIAN) Investigators. Circulation 2000;102:3032-8.

14. Zeymer U, Suryapranata H, Monassier JP, et al. The $\mathrm{Na}(+) / \mathrm{H}(+)$ exchange inhibitor eniporide as an adjunct to early reperfusion therapy for acute myocardial infarction.

Results of the evaluation of the safety and cardioprotective effects of eniporide in acute myocardial infarction (ESCAMI) trial. J Am Coll Cardiol 2001;38:1644-50.

15. McCully JD, Cowan DB, Pacak CA, et al. Injection of isolated mitochondria during early reperfusion for cardioprotection. Am J Physiol Heart Circ Physiol 2009;296:H94-105.

16. Masuzawa A, Black KM, Pacak CA, et al. Transplantation of autologously derived mitochondria protects the heart from ischemia-reperfusion injury. Am J Physiol Heart Circ Physiol 2013;304:H966-82.

17. Pacak CA, Preble JM, Kondo H, et al. Actin-dependent mitochondrial internalization in cardiomyocytes: evidence for rescue of mitochondrial function. Biol Open 2015;4:622-6.

18. Kaza AK, Wamala I, Friehs I, et al. Myocardial rescue with autologous mitochondrial transplantation in a porcine model of ischemia/reperfusion. J Thorac Cardiovasc Surg 2017;153:934-43.

19. Cowan DB, Yao R, Thedsanamoorthy JK, et al. Transit and integration of extracellular mitochondria in human heart cells. Sci Rep 2017;7:17450.

20. Cowan DB, Yao R, Akurathi V, et al. Intracoronary Delivery of Mitochondria to the Ischemic Heart for Cardioprotection. PLoS One 2016;11:e0160889.

21. Preble JM, Pacak CA, Kondo H, et al. Rapid isolation and purification of mitochondria for transplantation by tissue dissociation and differential filtration. J Vis Exp 2014;(91):e51682.

22. Preble JM, Kondo H, Levitsky S, et al. Quality Control Parameters for Mitochondria Transplant in Cardiac Tissue. JSM Biochem Mol Biol 2014;2:1008. 
23. Emani SM, Piekarski BL, Harrild D, et al. Autologous mitochondrial transplantation for dysfunction after ischemia-reperfusion injury. J Thorac Cardiovasc Surg 2017;154:286-9.

24. McCully JD, Levitsky S, Del Nido PJ, et al. Mitochondrial transplantation for therapeutic use. Clin Transl Med 2016;5:16.

25. Nasr VG, Faraoni D, DiNardo JA, et al. Association of Hospital Structure and Complications With Mortality After Pediatric Extracorporeal Membrane Oxygenation. Pediatr Crit Care Med 2016;17:684-91.

26. Chang JC, Wu SL, Liu KH, et al. Allogeneic/xenogeneic transplantation of peptide-labeled mitochondria in

Cite this article as: Emani SM, McCully JD. Mitochondrial transplantation: applications for pediatric patients with congenital heart disease. Transl Pediatr 2018;7(2):169-175. doi: 10.21037/tp.2018.02.02
Parkinson's disease: restoration of mitochondria functions and attenuation of 6-hydroxydopamine-induced neurotoxicity. Transl Res 2016;170:40-56.e3.

27. Flaquer A, Baumbach C, Ladwig KH, et al. Mitochondrial genetic variants identified to be associated with posttraumatic stress disorder. Transl Psychiatry 2015;5:e524.

28. Hayakawa K, Esposito E, Wang X, et al. Transfer of mitochondria from astrocytes to neurons after stroke. Nature 2016;535:551-5.

29. Lin HC, Liu SY, Lai HS, et al. Isolated mitochondria infusion mitigates ischemia-reperfusion injury of the liver in rats. Shock 2013;39:304-10. 